\title{
Educational needs and workplace opportunities of allied health assistants: A qualitative pilot
}

\author{
S. Mickan, X. Golenko \& N. Buys
}

\begin{abstract}
Introduction: Healthcare assistants perform a broad range of clinical and administrative tasks across many clinical settings and are supervised by most professional groups. This diversity creates challenges for maintaining a consistent scope of practice and consistent patterns of skill utilisation. It is not clear whether formal education in universities for allied health assistants (AHAs) could better shape the role and opportunities for the AHA workforce.

Methods and analysis: An exploratory qualitative study was designed to investigate the perspectives of senior allied health clinicians and academics about the educational needs and workplace opportunities for AHAs. We were interested in whether university education for AHAs could help to shape consistent roles and expectations of AHAs for the future workforce. A convenience sample of participants was invited to exploratory semi-structured interviews, and their responses were thematically analysed and integrated.

Results: Twelve participants, representing eight different allied health professions, were interviewed. Three themes emerged. Participants described the diverse roles, capabilities and expectations of the health assistant workforce. Current vocational training was considered inconsistent and insufficient, and participants reported significant local training occurring to meet the expectations of different work environments. Future educational pathways in university were not supported by any participants for many, largely practical, reasons.
\end{abstract}

Griffith Health, Gold Coast Campus, Griffith University

\author{
Correspondence \\ Sharon Mickan \\ Griffith Health, Gold Coast Campus \\ Griffith University \\ Queensland 4222 \\ Australia \\ Tel: +61 756780866 \\ Email: s.mickan@griffith.edu.au
}


Conclusion: University-level education does not appear to be a feasible tool to ensure consistent workforce roles and expectations for allied health assistants. Instead, AHAs need integrated educational pathways across vocational training and workplace environments. Continued workforce design is required to align assistant and professional roles and expectations.

Keywords: allied health professional; healthcare assistants; vocational training; educational needs assessment; professional education.

\section{Introduction}

Healthcare services globally are being challenged to respond to advances in technology and changing population profiles. Patients have greater access to information, which in turn, shapes their expectations and ability to participate in their healthcare decisions. Further, with an ageing population, current systems cannot sustain the management of chronic and complex diseases in hospital-based care. Consequently, healthcare systems and funding arrangements are changing, along with the practice and expectations of healthcare workers (Pearce \& Pagett, 2015). The shortage of health professionals in Australia has prompted workforce redesign initiatives and shifting boundaries between health professionals (Duckett, 2005; Nancarrow \& Borthwick, 2005; Stanhope \& Pearce, 2013). Medical specialists are sharing first contact with outpatients with extended-scope allied health professionals (AHPs) and nurse practitioners. With appropriate systems and supports, these extended-scope roles meet patient expectations for safe, effective and efficient care in areas of workforce shortage (Turnbull et al., 2009). At the same time, AHPs are allocating tasks to allied health assistants (AHAs) on the premise that the use of AHAs can help better manage demands on allied health services, improve patient outcomes and more efficiently maximise the skills of the workforce (Nancarrow, Roots, Grace, Moran, \& Vanniekerk-Lyons, 2013; Stute, Hurwood, Hulcombe, \& Kuipers, 2013).

Many AHPs supervise AHAs and delegate work with adults and children across a full range of clinical conditions and environments, with AHAs providing direct client care and indirect support. The actual range and type of clinical and administrative duties vary according to local clinical teams, their structures and processes. Clinical duties include administering clinical services, preparing patients and providing patient education and supervision. Administrative duties include preparing clinical environments, maintaining equipment, monitoring databases and housekeeping (Lizarondo, Kumar, Hyde, \& Skidmore, 2010). The use of AHAs has been reported to reduce the caseload burden of AHPs and create more time for them to concentrate on complex tasks (O'Brien, Byrne, Mitchell, \& Ferguson, 2013). AHAs are well accepted by clients, who appreciate the additional therapy time they receive. Healthcare benefits from using health assistants include increased intensity of clinical care, optimised patient flow, better professional skill usage and increased patient satisfaction (Lizarondo et al., 2010; O'Brien et al., 2013; Stute, Hurwood, Hulcombe, \& Kuipers, 2014).

Despite multiple initiatives across Australia to expand the scope of practice for AHPs and, consequently, increase opportunities to delegate to the AHA workforce, there has 
been limited consistency with the uptake of AHA roles (Stanhope \& Pearce, 2013; Stute et al., 2013). The diversity of tasks and work settings of AHPs, and therefore AHAs, provides challenges in defining and ensuring consistency in regards to scope of practice, skill utilisation and patterns of patient care responsibilities (Stanhope \& Pearce, 2013). Multiple studies have concluded that AHA roles need further clarity in order to promote better utilisation (Lizarondo et al., 2010; O'Brien et al., 2013; Pearce \& Pagett, 2015; Stute et al., 2014). Limited role clarity has also contributed to the inefficient use of AHAs in new models of care projects (Nancarrow et al., 2013). A recent Victorian workforce redesign study identified that almost $24 \%$ of the work completed by AHPs could be delegated to an AHA in community and ambulatory settings (Somerville, Davis, Milne, Terrill, \& Philip, 2017). This was reported across 12 of the 14 participating allied health professions, with the greatest opportunities being identified for podiatry, prosthetics/orthotics, speech pathology, nutrition and dietetics and social work professionals. The provision of clinical treatment and administrative tasks were identified as the most commonly performed tasks by AHAs in community and ambulatory settings. Delegation is also a documented challenge for AHPs, as it depends on the professional's confidence in the clinical skills of AHAs and their own ability to delegate tasks (Stute et al., 2014).

It is unclear whether the current educational training and qualifications of AHAs are sufficient to clarify roles and maximise AHAs contribution to the workplace. For many health professions, there has been a pattern of university-level qualifications serving as a prerequisite for defining a health professional's scope of practice, which is then reinforced through strict workplace entry requirements (Nancarrow \& Borthwick, 2005). For traditional Australian AHPs, undergraduate degree education has largely guaranteed their employment in Australia (Turnbull et al., 2009). This pattern does not currently exist for AHAs, as there are multiple qualifications, which are not always required as entry to the workplace. In Australia, the Certificates III and IV in Allied Health Assistance are reported as the preferred formal qualification for AHAs (Stute et al., 2014). However, this is often supplemented by significant informal training in the workplace. It is, therefore, unclear whether any further refinement of education standards for AHAs can shape consistent roles for the AHA workforce.

For all healthcare providers to be able to implement participative models of healthcare with patients and their families, Australia needs an efficient and coordinated interdisciplinary healthcare workforce to deliver evidence-based care in suitable locations. There is a need to match healthcare workers with appropriate tasks based on their skills and experience. Systems will be required to ensure all staff, including AHAs have appropriate skills, and appropriate mechanisms are needed to delegate and coordinate care between healthcare workers. Therefore, an opportunity exists to investigate whether the education and training of AHAs is sufficient for them to meet and maintain the workforce requirements of their role (Wallace, 2016). 


\begin{abstract}
Aim
This qualitative exploratory study was designed to investigate the perspectives of senior allied health clinicians and academics about the educational needs and workplace opportunities for AHAs. We were interested in whether university education for AHAs could help to shape consistent roles and expectations for the future AHA workforce.
\end{abstract}

\title{
Methods and analysis
}

This small explorative qualitative study was undertaken across two settings within Queensland: Griffith University and Queensland Health. Each setting represents a large key stakeholder group - academic and teaching staff from allied health programmes and senior AHP clinicians, managers and policy makers. A convenience sampling strategy was used to obtain a sample of senior academic, clinical managerial and policy staff who had strategic and experiential expertise in teaching, supervising and advocating for AHAs to participate in an individual interview about the educational and training needs of AHAs. Senior academic staff who had administrative responsibility for teaching and learning across all allied health, medical and nursing disciplines were invited to participate. Professional directors, policy leaders and workforce development officers who had managerial and support roles with AHA staff were invited from all allied health professions in the one health service.

Participants were contacted by the researchers to arrange a suitable time and location for the interview. Interviews were conducted face to face with both the principal investigator (PI) and co-investigator (CI) present. Interviews were audio-recorded, and notes were taken during the interview, with an interview template with a small set of open-ended questions sent to participants before the scheduled interview. Questions were grouped around four discussion areas: AHA role clarity, current support and supervision requirements, critique of current formal and informal education and outstanding educational needs. The interviews were semi-structured to allow for conversation and discussion around additional topics that participants considered important (Fylan, 2005). All interviews were recorded and independently transcribed, and participants were invited to review and edit their transcripts. Data were coded and categorised for comparison between stakeholder groups_clinicians and academics - to achieve an integrated perspective across all stakeholder groups. Thematic analysis was conducted in three stages (Castro, Kellison, Boyd, \& Kopak, 2010). First-level coding involved categorising descriptive verbal responses to the same questions across all participants. Second-level coding grouped similar responses within the four discussion areas. Finally, overarching themes emerged. Both researchers verified the first-level descriptive responses, second-level coding and third-level emergent themes (Eisner, 1998).

Ethical approval was granted by Griffith University. All participants provided written consent. 


\section{Results}

From the 22 academic and clinical staff invited to participate, 12 were interviewed. Participants represented six academic and six clinical workplaces. The professions represented at the interviews included speech pathology, dietetics, social work, physiotherapy, pharmacy, occupational therapy, psychology and rehabilitation counselling. Interviews were conducted in person and ranged from 30-60 minutes in length.

Three themes emerged, representing an integrated response to the original research question: (1) diverse roles, capabilities and expectations, (2) inconsistent prerequisite qualifications and training opportunities and (3) limited educational pathways. Each theme is described below, and italicised quotes are used to illustrate the perspectives of key stakeholders.

\section{Diverse roles, capabilities and expectations}

All participants described a broad range of roles and workplace expectations. Overall, clinician participants reported that health assistants were poorly paid and generally performed basic routine tasks to free up health professionals to perform more complex tasks. Participants described variations within AHA roles and their perceived contribution to the professional workforce. Examples of this variation included the report that some professionals "just let them do admin or manage the equipment" (Clinician 1), while other participants described situations where the AHAs' skills or capabilities were not being fully utilised: "I don't know that those therapy assistants necessarily get to use all that learning that they acquire" (Clinician 3).

Clinician participants raised concerns about a lack of consistent scope of practice for AHAs. Commonly, they attributed this to a lack of role clarity and reported that AHA roles were shaped by the patterns of local workplaces: "Some assistants are used to do photocopying and admin work and tasks that administration officers perhaps should be doing" (Clinician 5). Further, clinician participants reported that it was not always clear to health professionals whether AHAs had the skills and capabilities required for tasks delegated to them. Some clinician participants described how health assistants were not valued and were treated as "the bottom of the heap" (Clinician 2). Conversely, participants indicated that some AHAs represent a stable, older workforce who have significant experience and are extremely capable of doing routine tasks unsupervised: "Some assistants have been here a very long time, so their reputation is very positive. They're seen as the expert and they're well respected" (Clinician 4).

Clinician managers described having observed their professional peers' reluctance to delegate to AHAs unless there were longstanding relationships of trust and they knew AHAs had the required capability. While stable and older AHAs were noted to be competent and well respected, some AHAs were delegated basic routine tasks that did not fully utilise their capabilities. Managers also acknowledged that some professional staff did not have the skills to delegate tasks appropriately. Delegating to assistants was noted as especially difficult for new graduates, who were still trying to establish their identity and 
place within the profession and their workplace. Further, the assistants usually had more workplace experience than the new graduates.

Academic participants suggested that the assistant workforce may be perceived as a threat to the professions, where some professionals may be reluctant to let go of routine tasks for fear of losing recognition for some part of their role: "I understand that can undermine the professional position of the discipline" (Academic 2). Participants described situations where professionals did not have control over the assistants' scope of practice or their professional development and suggested this enhanced the perceived threat. It was suggested that this negative perception may be more pronounced within the acute setting. Participants generally reported that the assistant workforce played an important role in health settings such as community, remote, Indigenous, disability, rehabilitation, mental health and aged-care health services.

Finally, some academic participants indicated that the actual role of health assistants may be under threat due to advances in technology. They described the increased use of automation and robot technology and suggested that routine and predictable tasks will be able to be performed by computers and machines in the future. Given that many of the tasks performed by AHAs are routine and predictable, participants suggested that the role of allied health assistants may need to change in the future to remain viable and sustainable.

\section{Inconsistent prerequisite qualifications and training opportunities}

Participants recognised that formal training for the health assistant workforce is currently offered at the Certificate III or Certificate IV level through the vocational education and training (VET) sector. This includes technical and further education (TAFE) and private colleges, professional associations and online training packages within Queensland Health. Although many AHAs had a Certificate III or IV, clinician participants reported that formal training and qualifications were not a specified requirement for employment of AHAs in entry-level positions. While a Certificate IV was required for an advanced AHA, clinician participants reported that most hospital positions were not funded for these advanced positions. Further, clinician participants reported the inadequacy of formal education for local role expectations. They reported that AHAs required a high level of practical on-the-job training to be prepared for the practical tasks they were required to do: "It doesn't make them work ready ... [they are not able] to do any of the technical tasks we need them to do" (Clinician 6). Most commonly, participants described the provision of informal training in the workplace to meet practical and local expectations. However, clinician participants described limited opportunities for ongoing professional development and career advancement opportunities for their assistant health workforce. They described ways in which health assistants have not been supported.

They're not necessarily supported to go and do a lot of the training. They're not given the same opportunities and the professional development allowances to go and do that [with] the organisation supporting them and paying them to do it. (Clinician 2) 


\section{Limited educational pathways}

Participants confirmed that there are no current university qualifications for AHAs. Further, they did not support universities developing diploma-level qualifications for the assistant workforce. Clinician participants considered it unlikely that the financial costs of university study would be sufficiently reimbursed. They anticipated that employers were unlikely to support the high cost of a university qualification or offer higher pay levels for individuals after graduation. Clinician participants expressed concern that any increase in AHA pay or capability would conflict with new graduate AHPs in both role and costs, and this would reinforce managers' preference to employ new AHP graduates. A number of clinician participants indicated they preferred to employ new AHP graduates over assistants, especially when organisations were short-staffed due to budget constraints: "[New AHP graduates] skill up really quickly and have got so many other attributes that are valuable" (Clinician 5).

Further, both academic and clinical participants suggested that the high proportion of local and technical training for assistants needs to occur in the work environment and that it would be difficult for universities to deliver this vocational training. They emphasised that the ambiguity and instability around the health assistant role within the workplace and the lack of support from the professions presented a significant risk for university investment.

In some professions, assistant work experience was reported to prepare AHAs for professional qualifications: "There's a pathway that could be explored and maybe it's about setting up bridging pathways" (Academic 3). As an example, assistants who have worked in their role for a period of time were reported to move into professional degrees:

Mature-aged students that have been working around the community sector for a long time ... reach a point where they understand that they're probably being exploited financially, and if they come back and do a professional qualification, they'll be able to shift into higher positions and do different types of work that they probably aren't able or allowed to do. (Academic 3)

However, participants from other academically-competitive professions reported that the assistant workforce generally did not have the skills, capabilities or capacity to undertake higher-level studies: "I think some of them might struggle academically to achieve [a professional degree]" (Clinician 5). Academic participants reported that for some allied health professions, universities can fill their places with capable school leavers. Therefore, they suggested there is no need to recognise assistant qualifications as a pathway into a professional degree, and it may be a major disincentive for AHAs to start a full 4-year degree without any recognition of or credit for their assistant qualifications or work experience.

\section{Discussion}

This qualitative exploration of the educational needs and workplace opportunities for AHAs revealed three integrated themes across both academic and clinician participants. Firstly, the current diversity in roles, capabilities and expectations of AHAs is reflected 
in a broad range of contributions and perceived value within the workplace. These inconsistencies appear to limit the realisation of the full potential of AHAs in the workplace. Negative consequences are reinforced through a lack of clarity about AHA capabilities, limited successful delegation and some perceptions of role threat by health professionals.

Secondly, inconsistent prerequisite qualifications and training opportunities for the health assistant workforce were widely reported. While vocational training is preferred, it is not required for employment at entry level nor perceived by clinician managers as sufficient preparation. Most workplaces offer practical training, focused on the skills required in the local clinical context.

Thirdly, there is limited support for university educational pathways for the health assistant workforce. It was recognised that the high costs of university education are unlikely to be supported by employers, and it was considered unlikely that universities could support the high levels of practical training and support that AHAs required in local clinical settings. Although assistant work can prepare some AHAs for professional qualifications, this was not supported in all professions.

The overarching pattern of inconsistent AHA roles and capabilities appears to reinforce the diversity of views about the contribution and value of AHAs in the workplace. Further, it seems that employers' acceptance of multiple or no formal qualification serves to reinforce the variability of AHA roles and capabilities. In addition, local workplace training appears to shape local and unique patterns of AHAs' skills and capabilities, which may also reinforce the selective use of AHAs' skills. This pattern of limited role delineation has been reported to lower morale and workplace respect and hinder effective role development and utilisation (Stute et al., 2014). In this situation, it is unlikely that university education for AHAs will enhance consistent and appropriate utilisation of AHAs in the workforce.

There are inherent workforce challenges that will need to be addressed by clinicians and managers before education can be used to promote consistent roles and responsibilities. Continuing workplace reform and development is required to clarify and distinguish AHAs scope of practice so that it is integrated with redesigned roles of allied health professions. It will be important that both the health assistant and professional workforce are matched to appropriate tasks to maximise their contribution to patient care and to promote individual job satisfaction (Somerville at el., 2017). In addition, clear and consistent AHA roles and skills are required before educational opportunities can adequately prepare AHAs for their work roles (Stute et al., 2014; Turnbull et al., 2009). This would ensure AHAs are integrated into teams with their supervising AHPs to meet the current and future healthcare needs of the broader population in an efficient and sustainable manner (Somerville et al., 2017).

Requirements for formal training are likely to translate to expectations of higher wages (Tache \& Chapman, 2006). This could be problematic for AHAs in Australia. The public sector wage structures for AHPs provide little recognition of clinical experience or academic training (Turnbull et al., 2009). It is unlikely that income levels would change for AHAs if they had higher levels of formal education. 


\section{Limitations and recommendations}

A major limitation of this study was its small convenience sample, which may limit the applicability of reported results to other locations and health settings.

Future recommendations for the education of AHAs should include the development of clear training pathways that are integrated across vocational training and workplace environments and include components of multidisciplinary care. Training should complement workplace learning and development within a broader professional support framework (Somerville et al., 2017; Turnbull et al., 2009). Authentic engagement in and learning through consistent workplace practices can also serve to embed consistent identity and role expectations (Billett \& Somerville, 2004). It will be important to monitor AHPs skills and confidence in delegating to AHAs and monitor AHPs satisfaction with working with AHAs as potential outcomes of an effective educational system (Somerville et al., 2017).

\section{Conclusion}

Despite the potential for AHAs to release time for professionals to fulfil and extend their scope of practice, there are workforce concerns about a lack of consistent roles and expectations, coupled with limited delegation capability by professionals. The current diversity and consequent inconsistencies in AHA roles, capabilities and expectations have limited their contribution to and perceived value within the workplace. Concurrently, inconsistent educational requirements have reinforced local variations in skills and practical training. There is also limited support for university educational pathways for the health assistant workforce. The high costs of university education are unlikely to be supported by employers, and universities are unlikely to be able to deliver the high levels of practical training and support that AHAs require in local clinical settings. Instead, AHAs need integrated educational pathways across vocational training and workplace environments. Continued workforce redesign is required to align assistant and professional roles and expectations.

\section{Acknowledgements}

We would like to thank all participants for their time and valuable insights.

\section{Funding and conflict of interest}

No conflict of interest exists for any author.

\section{References}

Billett, S., \& Somerville, M. (2004). Transformations at work: Identity and learning. Studies in Continuing Education, 26(2), 309-326. doi:10.1080/158037042000225272

Castro, F. G., Kellison, J. G., Boyd, S. J., \& Kopak, A. (2010). A methodology for conducting integrative mixed methods research and data analyses. Journal of Mixed Methods Research, 4(4), 342-360. doi:10.1177/1558689810382916 
Duckett, S. J. (2005). Interventions to facilitate health workforce restructure. Australia and New Zealand Health Policy, 2(14), 1-6.

Eisner, E. W. (1998). The enlightened eye: Qualitative enquiry and the enhancement of educational practice. Upper Saddle River, NJ: Prentice Hall.

Fylan, F. (2005). Semi-structured interviewing. In J. Miles \& P. Gilbert (Eds.), A handbook of research methods for clinical and health psychology (pp. 65-78). New York, NY: Oxford University Press.

Lizarondo, L., Kumar, S., Hyde, L., \& Skidmore, D. (2010). Allied health assistants and what they do: A systematic review of the literature. Journal of Multidisciplinary Healthcare, 3, 143-153. doi:10.2147/JMDH.S12106

Nancarrow, S. A., \& Borthwick, A. M. (2005). Dynamic professional boundaries in the healthcare workforce. Sociology of Health \& Illness, 27(7), 897-919. doi:10.1111/j.1467-9566.2005.00463.x

Nancarrow, S. A., Roots, A., Grace, S., Moran, A. M., \& Vanniekerk-Lyons, K. (2013). Implementing large-scale workforce change: Learning from 55 pilot sites of allied health workforce redesign in Queensland, Australia. Human Resources for Health, 11(1), 66. doi:10.1186/1478-4491-11-66

O’Brien, R., Byrne, N., Mitchell, R., \& Ferguson, A. (2013). Rural speech-language pathologists' perceptions of working with allied health assistants. International Journal of Speech-Language Pathology, 15(6), 613-622.

Pearce, C., \& Pagett, L. (2015). Advanced allied health assistants: An emerging workforce. Australian Health Review, 39(3), 260-263. doi:10.1071/AH14253

Somerville, L., Davis, A., Milne, S., Terrill, D., \& Philip, K. (2017). Exploration of an allied health workforce redesign model: Quantifying the work of allied health assistants in a community workforce. Australian Health Review. Advance online publication. doi:10.1071/AH16266

Stanhope, J., \& Pearce, C. (2013). Role, implementation, and effectiveness of advanced allied health assistants: A systematic review. Journal of Multidisciplinary Healthcare, 6, 423-434. doi:10.2147/JMDH.S50185

Stute, M., Hurwood, A., Hulcombe, J., \& Kuipers, P. (2013). Defining the role and scope of practice of allied health assistants within Queensland public health services. Australian Health Review, 37(5), 602-606. doi:10.1071/AH13042

Stute, M., Hurwood, A., Hulcombe, J., \& Kuipers, P. (2014). Pilot implementation of allied health assistant roles within publicly funded health services in Queensland, Australia: Results of a workplace audit. BMC Health Services Research, 14(1), 258. doi:10.1186/1472-6963-14-258

Taché, S., \& Chapman, S. (2006). The expanding roles and occupational characteristics of medical assistants: Overview of an emerging field in allied health. Journal of Allied Health, 35(4), 233-237.

Turnbull, C., Grimmer-Somers, K., Kumar, S., May, E., Law, D., \& Ashworth, E. (2009). Allied, scientific and complementary health professionals: A new model for Australian allied health. Australian Health Review, 33(1), 27-37. 
EDUCATIONAL NEEDS AND WORKPLACE OPPORTUNITIES OF ALLIED HEALTH ASSISTANTS

Wallace, S. (2016). Re: Building allied health workforce capacity: A strategic approach to workforce innovation [Comment]. Australian Health Review, 40(4), 473. doi:10.1071/AH15176 